\title{
Ureido Derivatives of Neoabietic Acid
}

\author{
Xinyu Gao ${ }^{1,2}$, Niping Feng ${ }^{1}$, Yuhan $\mathrm{Zi}^{1}$, Jianguo Cao ${ }^{1, *}$ and Guozheng Huang $1,3, * \mathbb{C}$ \\ 1 College of Life and Environmental Sciences, Shanghai Normal University, Shanghai 201418, China; \\ xinyugao@126.com (X.G.); fengniping@163.com (N.F.); zizizi1994@126.com (Y.Z.) \\ 2 College of Mathematics, Physics and Information Engineering, Zhejiang Normal University, \\ Jinhua 321004, China \\ 3 Key Laboratory of Plant Resources and Chemistry of Arid Zone, State Key Laboratory Basis of Xinjiang \\ Indigenous Medicinal Plants Resource Utilization, Xinjiang Technical Institute of Physics and Chemistry, \\ Chinese Academy of Sciences, Urumqi 830011, China \\ * Correspondence: cao101@shnu.edu.cn (J.C.); guozheng.huang@yahoo.com or g.huang@ms.xjb.ac.cn (G.H.); \\ Tel.: +86-21-64322526 (J.C.); +86-991-3836733 (G.H.)
}

Received: 14 November 2018; Accepted: 26 November 2018; Published: 29 November 2018

\begin{abstract}
A series of ureido derivatives of neoabietic acid were synthesized by application of Curtius rearrangement reaction to neoabietic acid and amines. Structure characterization of these compounds was done by ${ }^{1} \mathrm{H}-\mathrm{NMR},{ }^{13} \mathrm{C}-\mathrm{NMR}$ and HRMS spectral analysis.
\end{abstract}

Keywords: neoabietic acid; urea; structural modification

\section{Introduction}

Resin acids are dozens of natural monoterpene, sesquiterpene and diterpene acids found in the oleoresin which was secreted by the plants to treat against herbivores and other pathogens. When attacked by herbivores, such as white pine weevil, bark beetle, pine sawfly and so forth, or fungus and bacteria, the plants (mostly of Pinaceae family) can produce a mixture of viscous compounds containing resin acids to push out or entomb the attacking insects, and/or seal and heal the wounding [1]. Chemically, resin acids contain a mixture of isomeric abietic-type acids and pimaric-type acids. Abietic, neoabietic, dehydroabietic, levopimaric and palustric acids are the commonly found resin acids in nature (Figure 1). Outside the natural contexts, biological activities of resin acids were also well studied. For example, abietic acid was found to enhance cell migration and tube formation in human umbilical vein vascular endothelial cells, by upregulation of extracellular signal-regulated kinase and p38 expression [2]. Fox et al. found that abietic acid can inhibit protein tyrosinephosphatase $1 \mathrm{~B}$, which might be of benefit to the treatment of diseases related to insulin signaling [3]. It is discovered that the oral administration of methyl dehydroabietate can reduce white adipose tissue weight and plasma levels of glucose, insulin and leptin in mice induced by high-fat $\operatorname{diet}[4]$.<smiles>CC(C)C1=CC2=CC[C@H]3[C@@](C)(C(=O)O)CCC[C@]3(C)C2CC1</smiles>

abietic acid<smiles>CC(C)C1=CC2=C(CC1)[C@]1(C)CCC[C@](C)(C(=O)O)[C@@H]1CC2</smiles>

palustric acid<smiles>CC(C)c1ccc2c(c1)CC[C@H]1[C@@](C)(C(=O)O)CCC[C@@]21C</smiles>

dehydroabietic acid

Figure 1. Structure of representative resin acids. 
The diverse biological potentials inspired chemically structural modification of resin acids [5]. Recently Hou et al. synthesized a dozen amides based on dehydroabietic acid and evaluated their antibacterial activity [6]. Other derivatives of resin acids were also prepared [7-9]. Nevertheless, synthetic modification has not yet been pursued up to now regarding to neoabietic acid.

Curtius rearrangement reaction is a classic organic reaction for preparation of carbamates, amines and urea derivative from acyl azides, which could be easily synthesized from carboxylic acids [10]. Curtius rearrangement reaction has recently used to steviol, a naturally occurring diterpenoid to obtain potent inhibitor against hepatitis B virus [11]. We assume the similar strategy could be applied to neoabietic acid and herein we reported the synthesis and structural characterization of ureido derivatives of neoabietic acid.

\section{Results and Discussion}

The synthesis of C-4 ureido compounds of neoabietic acid was depicted in Scheme 1. Generally, abietic acid was dissolved in toluene, followed by the addition of diphenylphosphoryl azide (DPPA) and triethylamine. The mixture was heated at $110^{\circ} \mathrm{C}$ to be completely converted into the isocyanate. Without isolation of the isocyanate, to the cooled reaction mixture was added the respectful amine. The final target urea derivatives were usually formed after stirring at $80^{\circ} \mathrm{C}$ for $4-10 \mathrm{~h}$.

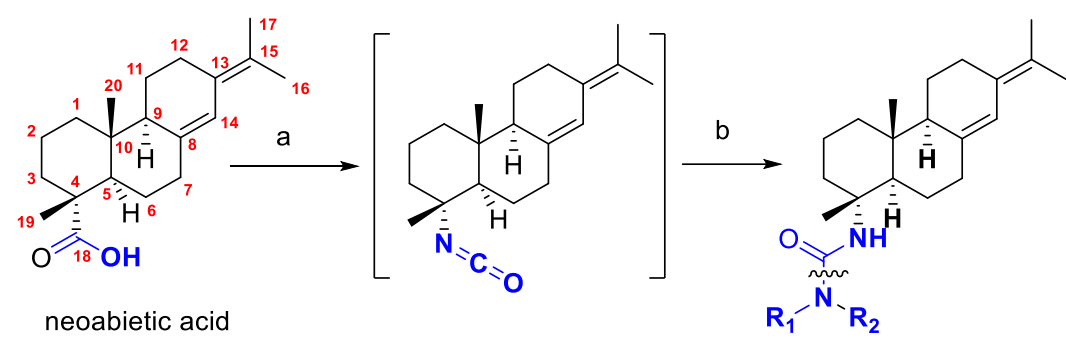

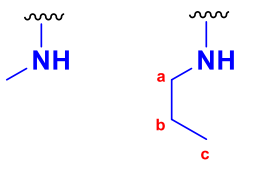

1<smiles>[Z7]C1CCN(C2CCN(C(C)(C)C)CC2)CC1</smiles>

2<smiles>CN1CCC(N(C)C)CC1</smiles>

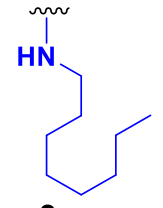

3<smiles>CN1CCC(N2CCOCC2)CC1</smiles>

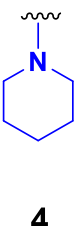

4<smiles>CNc1ccccc1O</smiles>

10

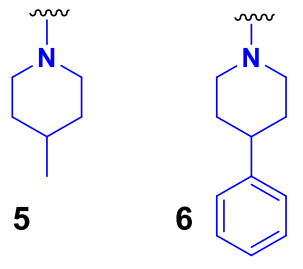<smiles>CNCCc1c[nH]c2ccccc12</smiles>

11

Scheme 1. Synthesis of ureido derivatives of neoabietic acid. Reagents and condition: (a): DPPA, triethylamine, toluene, $110{ }^{\circ} \mathrm{C}, 2-4 \mathrm{~h} ;(\mathbf{b})$ amine, toluene, $80^{\circ} \mathrm{C}, 4-10 \mathrm{~h}$.

Lin et al. isolated the intermediate isocyanate when preparing steviol ureido derivatives [11], that is, $4 \alpha$-Isocyanato-19-nor-ent-13-hydroxykaur-16-ene, as a white crystal. In our case, attempt to purify the intermediate proved fruitless. Thus, we resorted to the one-pot, two-step protocol for the synthesis of compounds 1-11. This strategy is tolerable to a broad range of aliphatic primary and secondary amine but is not applicable to aromatic amines with electron-withdrawing groups. For example, reaction of isocyanate intermediate with 4-methylaniline, 4-chloroaniline, 
2-chloropyridin-3-amine, 4-methylpyridin-2-amine, did not obtain any desired product. Nevertheless, 2-aminophenol was successfully be converted to the urea compound $\mathbf{1 0}$.

It is observed that the synthesized ureido derivatives of neoabietic acid are stable in neutral and basic condition but acidic mediate would lead to their slow decomposition.

\section{Materials and Methods}

Commercially available reagents were used as received without additional purification. Thin-layer chromatography (TLC) was carried out on glass plates coated with silica gel (Qingdao Haiyang Chemical Co., Qingdao, China, G60F-254) and visualized by UV light (254 nm). The products were purified by column chromatography over silica gel (Qingdao Haiyang Chemical Co., Qingdao, China, 200-300 mesh). NMR spectra were recorded with a Varian $400 \mathrm{MHz}$ or a $500 \mathrm{MHz}$ NMR spectrometer in $\mathrm{CDCl}_{3}, \mathrm{DMSO}-d_{6}$ or pyridine- $d_{5}$. High-resolution mass spectra (HRMS, electrospray in positive mode, ESI+) were recorded on AB SCIEX Elite quadrupole time-of-flight mass spectrometer.

General procedure for the preparation of C-4 ureido compounds 1-11: To the solution of neoabietic acid $(30.2 \mathrm{mg}, 0.1 \mathrm{mmol})$ in toluene $(3 \mathrm{~mL})$, were added diphenylphosphoryl azide ( $35.8 \mathrm{mg}, 0.13 \mathrm{mmol})$ and triethylamine $(13 \mathrm{mg}, 0.13 \mathrm{mmol})$. The mixture was heated at $110{ }^{\circ} \mathrm{C}$ for $2-4 \mathrm{~h}$. After TLC confirmed the total consumption of neoabietic acid, the reaction mixture was then cooled to $80^{\circ} \mathrm{C}$ and charged with amine $(0.15 \mathrm{mmol})$. The resulting mixture was stirred at $80^{\circ} \mathrm{C}$ for another $4-10 \mathrm{~h}$ before quenched with water. The solvents were evaporated off in vacuo, the residue was purified by column chromatography to produce the desired product.

1-[(1R,4aR,4bS,10aR)-1,4a-Dimethyl-7-(propan-2-ylidene)-1,2,3,4,4a,4b,5,6,7,9,10,10a-

dodecahydrophenanthren-1-yl]-3-methylurea (1), Yield 87\%, white solid. ${ }^{1} \mathrm{H}-\mathrm{NMR}\left(500 \mathrm{MHz}\right.$, pyridine- $\left.d_{5}\right)$ $\delta 6.33(\mathrm{~s}, 1 \mathrm{H}), 6.11(\mathrm{q}, J=4.8 \mathrm{~Hz}, 1 \mathrm{H}), 5.82(\mathrm{~s}, 1 \mathrm{H}), 2.88(\mathrm{~d}, J=4.7 \mathrm{~Hz}, 3 \mathrm{H}), 2.60-2.50(\mathrm{~m}, 2 \mathrm{H}), 2.42-2.28$ $(\mathrm{m}, 2 \mathrm{H}), 2.24-2.11(\mathrm{~m}, 2 \mathrm{H}), 1.95(\mathrm{~d}, J=8.7 \mathrm{~Hz}, 1 \mathrm{H}), 1.92-1.81(\mathrm{~m}, 2 \mathrm{H}), 1.75(\mathrm{~s}, 3 \mathrm{H}), 1.70(\mathrm{~s}, 3 \mathrm{H}), 1.62-1.50$ $(\mathrm{m}, 2 \mathrm{H}), 1.49-1.34(\mathrm{~m}, 4 \mathrm{H}), 1.31(\mathrm{~s}, 3 \mathrm{H}), 1.17(\mathrm{td}, J=12.9,3.7 \mathrm{~Hz}, 1 \mathrm{H}), 0.77(\mathrm{~s}, 3 \mathrm{H}) .{ }^{13} \mathrm{C}-\mathrm{NMR}(125 \mathrm{MHz}$, pyridine- $\left.d_{5}\right) \delta 159.29,139.52,129.33,123.44,123.05,56.56,52.14,51.61,39.58,39.16,39.09,36.45,27.27$, 26.73, 23.31, 23.12, 22.42, 20.87, 20.47, 20.22, 15.95. HRMS (ESI) calcd. for $\mathrm{C}_{21} \mathrm{H}_{35} \mathrm{~N}_{2} \mathrm{O}[\mathrm{M}+\mathrm{H}]^{+}$: 331.27439, found: 331.27429 .

1-[(1R,4aR,4bS,10aR)-1,4a-Dimethyl-7-(propan-2-ylidene)-1,2,3,4,4a,4b,5,6,7,9,10,10adodecahydrophenanthren-1-yl]-3-propylurea (2), Yield 82\%, white solid. ${ }^{1} \mathrm{H}-\mathrm{NMR}\left(400 \mathrm{MHz}, \mathrm{DMSO}-d_{6}\right) \delta$ $6.17(\mathrm{~s}, 1 \mathrm{H}, 14-\mathrm{CH}), 5.63(\mathrm{~s}, 1 \mathrm{H}, \mathrm{NH}), 5.40(\mathrm{~s}, 1 \mathrm{H}, \mathrm{NH}), 2.89-2.84\left(\mathrm{~m}, 2 \mathrm{H}, \mathrm{a}-\mathrm{CH}_{2}\right), 2.49-2.42(\mathrm{~m}, 1 \mathrm{H}$, 12- $\left.\mathrm{CH}_{2}\right), 2.38-2.30\left(\mathrm{~m}, 1 \mathrm{H}, 7-\mathrm{CH}_{2}\right), 2.12-2.00\left(\mathrm{~m}, 1 \mathrm{H}, 7^{\prime}-\mathrm{CH}_{2}\right), 1.98-1.90\left(\mathrm{~m}, 2 \mathrm{H}, 3\right.$ \& $\left.5-\mathrm{CH}_{2}\right), 1.88-1.70$ $\left(\mathrm{m}, 4 \mathrm{H}, 2,3^{\prime}, 9 \& 12^{\prime}-\mathrm{CH}_{2}\right), 1.68\left(\mathrm{~s}, 3 \mathrm{H}, 16-\mathrm{CH}_{3}\right), 1.65\left(\mathrm{~s}, 3 \mathrm{H}, 17-\mathrm{CH}_{3}\right), 1.63-1.56\left(\mathrm{~m}, 2 \mathrm{H}, 1\right.$ \& 6- $\left.\mathrm{CH}_{2}\right)$, $1.49-1.42\left(\mathrm{~m}, 1 \mathrm{H}, 11-\mathrm{CH}_{2}\right), 1.39-1.24\left(\mathrm{~m}, 5 \mathrm{H}, 2^{\prime}, 6^{\prime}, \mathrm{b} \& 1^{\prime}-\mathrm{CH}_{2}\right), 1.09\left(\mathrm{~s}, 3 \mathrm{H}, 19-\mathrm{CH}_{3}\right), 1.06-0.94(\mathrm{~m}, 1 \mathrm{H}$, $\left.1^{\prime}-\mathrm{CH}_{2}\right), 0.82\left(\mathrm{t}, J=7.4 \mathrm{~Hz}, 3 \mathrm{H}, \mathrm{c}-\mathrm{CH}_{3}\right), 0.68\left(\mathrm{~s}, 3 \mathrm{H}, 20-\mathrm{CH}_{3}\right) .{ }^{13} \mathrm{C}-\mathrm{NMR}\left(100 \mathrm{MHz}, \mathrm{DMSO}-d_{6}\right) \delta 157.06$ (18-CO), 138.33 (8-C), 127.82 (13-C), 122.54 (15-C), 121.80 (14-C), 54.80 (4-C), 50.90 (9-C), 50.25 (5-C), 40.56 (a-C), 38.36 (10-C), 38.06 (3-C), 37.71 (1-C), 35.25 (7-C), 25.46 (12-C), 23.26 (b-C), 22.08 (2-C), 21.70 (6-C), 21.33 (19-C), 20.18 (17-C), 19.45 (16-C), 19.09 (11-C), 15.03 (20-C), 11.34 (c-C). HRMS (ESI) calcd. for $\mathrm{C}_{23} \mathrm{H}_{39} \mathrm{~N}_{2} \mathrm{O}[\mathrm{M}+\mathrm{H}]^{+}$: 359.3062, found: 359.3050, $717.6020\left\{[2 \mathrm{M}+\mathrm{H}]^{+}\right\}$.

1-[(1R,4aR,4bS,10aR)-1,4a-Dimethyl-7-(propan-2-ylidene)-1,2,3,4,4a,4b,5,6,7,9,10,10adodecahydrophenanthren-1-yl]-3-octylurea (3), Yield 75\%, white solid. ${ }^{1} \mathrm{H}-\mathrm{NMR}\left(500 \mathrm{MHz}, \mathrm{CDCl}_{3}\right) \delta 6.24$ $(\mathrm{s}, 1 \mathrm{H}), 4.13(\mathrm{~s}, 1 \mathrm{H}), 4.06(\mathrm{~s}, 1 \mathrm{H}), 3.12(\mathrm{q}, J=6.6 \mathrm{~Hz}, 2 \mathrm{H}), 2.55(\mathrm{dt}, J=14.1,4.2 \mathrm{~Hz}, 1 \mathrm{H}), 2.41(\mathrm{dd}, J=15.1$, $4.0 \mathrm{~Hz}, 1 \mathrm{H}), 2.30-2.14(\mathrm{~m}, 2 \mathrm{H}), 2.09(\mathrm{dd}, J=12.7,2.5 \mathrm{~Hz}, 1 \mathrm{H}), 2.03(\mathrm{t}, J=7.8 \mathrm{~Hz}, 1 \mathrm{H}), 1.98-1.80(\mathrm{~m}, 4 \mathrm{H})$, $1.74(\mathrm{~s}, 3 \mathrm{H}), 1.71(\mathrm{~d}, J=7.5,1 \mathrm{H}), 1.67(\mathrm{~s}, 4 \mathrm{H}), 1.61(\mathrm{dt}, J=14.1,3.7 \mathrm{~Hz}, 2 \mathrm{H}), 1.54-1.37(\mathrm{~m}, 5 \mathrm{H}), 1.36-1.26$ $(\mathrm{m}, 8 \mathrm{H}), 1.25(\mathrm{~s}, 3 \mathrm{H}), 1.23-1.19(\mathrm{~m}, 1 \mathrm{H}), 0.91(\mathrm{t}, J=6.7 \mathrm{~Hz}, 3 \mathrm{H}), 0.80(\mathrm{~s}, 3 \mathrm{H}) .{ }^{13} \mathrm{C}-\mathrm{NMR}(125 \mathrm{MHz}$, $\mathrm{CDCl}_{3}$ ) $\delta 157.16$ (18-CO), 138.79 (8-C), 128.49 (13-C), 123.48 (15-C), 122.17 (14-C), 56.70, 51.50, 51.15, $40.67,39.02,38.48,38.20,35.82,31.95,30.45,29.48,29.40,27.12,26.02,22.79,22.68,22.46,22.28,20.49$, 19.83, 19.70, 15.47, 14.23. HRMS (ESI) calcd. for $\mathrm{C}_{28} \mathrm{H}_{49} \mathrm{~N}_{2} \mathrm{O}[\mathrm{M}+\mathrm{H}]^{+}: 429.38394$, found: 429.38400 . 
$N-[(1 R, 4 a R, 4 b S, 10 a R)-1,4 a-D i m e t h y l-7-($ propan-2-ylidene)-1,2,3,4,4a,4b,5,6,7,9,10,10a-

dodecahydrophenanthren-1-yl]piperidine-1-carboxamide (4), Yield 69\%, colorless oil. ${ }^{1} \mathrm{H}-\mathrm{NMR}(500 \mathrm{MHz}$, $\left.\mathrm{CDCl}_{3}\right) \delta 6.18(\mathrm{~s}, 1 \mathrm{H}), 4.16(\mathrm{~s}, 1 \mathrm{H}), 3.24(\mathrm{t}, J=5.3 \mathrm{~Hz}, 4 \mathrm{H}), 2.50(\mathrm{dt}, J=14.1,4.4 \mathrm{~Hz}, 1 \mathrm{H}), 2.36(\mathrm{dd}, J=14.4$, $4.5 \mathrm{~Hz}, 1 \mathrm{H}), 2.20(\mathrm{qd}, J=13.6,12.6,3.9 \mathrm{~Hz}, 2 \mathrm{H}), 2.10(\mathrm{dd}, J=12.6,2.5 \mathrm{~Hz}, 1 \mathrm{H}), 1.99(\mathrm{~d}, J=8.0 \mathrm{~Hz}, 1 \mathrm{H})$, $1.93-1.75(\mathrm{~m}, 4 \mathrm{H}), 1.73(\mathrm{~s}, 3 \mathrm{H}), 1.68(\mathrm{~s}, 3 \mathrm{H}), 1.67-1.49(\mathrm{~m}, 8 \mathrm{H}), 1.47-1.28(\mathrm{~m}, 3 \mathrm{H}), 1.21(\mathrm{~s}, 3 \mathrm{H}), 1.16$ $(\mathrm{dd}, J=13.1,3.8 \mathrm{~Hz}, 1 \mathrm{H}), 0.75$ (s, 3H). ${ }^{13} \mathrm{C}-\mathrm{NMR}\left(125 \mathrm{MHz}, \mathrm{CDCl}_{3}\right) \delta 156.79,138.83,128.51,123.38$, $122.11,56.83,51.47,50.80,45.26\left(2 \times \mathrm{CH}_{2}\right), 39.00,38.43,37.91,35.82,26.01,25.81\left(2 \times \mathrm{CH}_{2}\right), 24.63$, 22.66, 22.51, 22.27, 20.45, 19.80, 19.71, 15.44. HRMS (ESI) calcd. for $\mathrm{C}_{25} \mathrm{H}_{41} \mathrm{~N}_{2} \mathrm{O}[\mathrm{M}+\mathrm{H}]^{+}: 385.32134$, found: 385.32138 .

N-[(1R,4aR,4bS,10aR)-1,4a-Dimethyl-7-(propan-2-ylidene)-1,2,3,4,4a,4b,5,6,7,9,10,10a-

dodecahydrophenanthren-1-yl]-4-methylpiperidine-1-carboxamide (5), Yield 72\%, colorless oil. ${ }^{1} \mathrm{H}-\mathrm{NMR}$ $\left(500 \mathrm{MHz}, \mathrm{CDCl}_{3}\right) \delta 6.19(\mathrm{~s}, 1 \mathrm{H}), 4.17(\mathrm{~s}, 1 \mathrm{H}), 3.85-3.77(\mathrm{~m}, 2 \mathrm{H}), 2.74-2.64(\mathrm{~m}, 2 \mathrm{H}), 2.51(\mathrm{dt}, J=14.2$, $4.4 \mathrm{~Hz}, 1 \mathrm{H}), 2.37(\mathrm{dd}, J=14.3,4.6 \mathrm{~Hz}, 1 \mathrm{H}), 2.27-2.14(\mathrm{~m}, 2 \mathrm{H}), 2.09(\mathrm{dd}, J=12.7,2.5 \mathrm{~Hz}, 1 \mathrm{H}), 1.99$ $(\mathrm{d}, J=8.2 \mathrm{~Hz}, 1 \mathrm{H}), 1.93-1.75(\mathrm{~m}, 4 \mathrm{H}), 1.73(\mathrm{~s}, 3 \mathrm{H}), 1.69(\mathrm{~s}, 3 \mathrm{H}), 1.69-1.50(\mathrm{~m}, 8 \mathrm{H}), 1.50-1.28(\mathrm{~m}, 4 \mathrm{H})$, $1.26-1.02(\mathrm{~m}, 4 \mathrm{H}), 0.93(\mathrm{~d}, J=6.4 \mathrm{~Hz}, 3 \mathrm{H}), 0.76(\mathrm{~s}, 3 \mathrm{H}) .{ }^{13} \mathrm{C}-\mathrm{NMR}\left(125 \mathrm{MHz}, \mathrm{CDCl}_{3}\right) \delta 156.79,138.83$, 128.51, 123.41, 122.13, 56.86, 51.47, 50.81, 44.70, 44.64, 39.00, 38.44, 37.91, 35.81, 34.12, 34.07, 31.12, $26.01,22.66,22.51,22.28,21.95,20.46,19.81,19.71,15.45$. HRMS (ESI) calcd. for $\mathrm{C}_{26} \mathrm{H}_{43} \mathrm{~N}_{2} \mathrm{O}[\mathrm{M}+\mathrm{H}]^{+}$: 399.33699, found: 399.33691 .

N-[(1R,4aR,4bS,10aR)-1,4a-Dimethyl-7-(propan-2-ylidene)-1,2,3,4,4a,4b,5,6,7,9,10,10a-

dodecahydrophenanthren-1-yl]-4-phenylpiperidine-1-carboxamide (6), Yield 80\%, colorless oil. ${ }^{1} \mathrm{H}-\mathrm{NMR}$ $\left(500 \mathrm{MHz}, \mathrm{CDCl}_{3}\right) \delta 7.31(\mathrm{t}, J=7.4 \mathrm{~Hz}, 2 \mathrm{H}), 7.20(\mathrm{~d}, J=7.9 \mathrm{~Hz}, 3 \mathrm{H}), 6.21(\mathrm{~s}, 1 \mathrm{H}), 4.24(\mathrm{~s}, 1 \mathrm{H}), 4.00$ $(\mathrm{t}, J=10.0 \mathrm{~Hz}, 2 \mathrm{H}), 2.89-2.78(\mathrm{~m}, 2 \mathrm{H}), 2.64(\mathrm{tt}, J=12.1,3.7 \mathrm{~Hz}, 1 \mathrm{H}), 2.52(\mathrm{dt}, J=14.0,4.3 \mathrm{~Hz}, 1 \mathrm{H}), 2.40$ $(\mathrm{dd}, J=14.6,4.3 \mathrm{~Hz}, 1 \mathrm{H}), 2.31-2.18(\mathrm{~m}, 2 \mathrm{H}), 2.14(\mathrm{dd}, J=12.7,2.5 \mathrm{~Hz}, 1 \mathrm{H}), 2.03(\mathrm{t}, J=7.9 \mathrm{~Hz}, 1 \mathrm{H}), 1.93$ $(\mathrm{d}, J=12.8 \mathrm{~Hz}, 1 \mathrm{H}), 1.88-1.82(\mathrm{~m}, 3 \mathrm{H}), 1.75(\mathrm{~s}, 3 \mathrm{H}), 1.70(\mathrm{~s}, 3 \mathrm{H}), 1.72-1.56(\mathrm{~m}, 6 \mathrm{H}), 1.53-1.30(\mathrm{~m}, 3 \mathrm{H})$, $1.25(\mathrm{~s}, 3 \mathrm{H}), 1.24-1.16(\mathrm{~m}, 1 \mathrm{H}), 0.78(\mathrm{~s}, 3 \mathrm{H}) .{ }^{13} \mathrm{C}-\mathrm{NMR}\left(125 \mathrm{MHz}, \mathrm{CDCl}_{3}\right) \delta 156.71,145.76,138.76,128.66$ $(2 \times \mathrm{ArCH}), 128.50,126.89(2 \times \mathrm{ArCH}), 126.51,123.47,122.19,57.00,51.49,50.83,45.16,45.08,42.86$, $39.03,38.45,37.92,35.83,33.28,33.21,26.02,22.68,22.57,22.31,20.48,19.82,19.72,15.46$. HRMS (ESI) calcd. for $\mathrm{C}_{31} \mathrm{H}_{45} \mathrm{~N}_{2} \mathrm{O}[\mathrm{M}+\mathrm{H}]^{+}$: 461.35264, found: 461.35253 .

N-[(1R,4aR,4bS,10aR)-1,4a-Dimethyl-7-(propan-2-ylidene)-1,2,3,4,4a,4b,5,6,7,9,10,10a-

dodecahydrophenanthren-1-yl]-[1,4'-bipiperidine]-1'-carboxamide (7), Yield 71\%, white solid. ${ }^{1} \mathrm{H}-\mathrm{NMR}$ $\left(400 \mathrm{MHz}, \mathrm{DMSO}-d_{6}\right) \delta 6.16(\mathrm{~s}, 1 \mathrm{H}), 5.37(\mathrm{~s}, 1 \mathrm{H}), 3.91(\mathrm{~d}, J=12.9 \mathrm{~Hz}, 2 \mathrm{H}), 2.58-2.48(\mathrm{~m}, 4 \mathrm{H}$, hiding in DMSO), 2.45-2.39 (m, 4H), 2.35-2.27 (m, 2H), 2.23-2.15 (m, 2H), 2.14-2.06 (m, 2H), 1.89-1.84 (m, 1H), $1.82-1.58(\mathrm{~m}, 12 \mathrm{H}), 1.49-1.43(\mathrm{~m}, 4 \mathrm{H}), 1.39-1.34(\mathrm{~m}, 2 \mathrm{H}), 1.31-1.18(\mathrm{~m}, 4 \mathrm{H}), 1.13(\mathrm{~s}, 3 \mathrm{H}), 1.09-1.04(\mathrm{~m}$, $1 \mathrm{H}), 0.73(\mathrm{~s}, 3 \mathrm{H}) .{ }^{13} \mathrm{C}-\mathrm{NMR}\left(100 \mathrm{MHz}, \mathrm{DMSO}-d_{6}\right) \delta 156.52$ (18-CO), 138.51 (8-C), 127.84 (13-C), 122.47 (15-C), 121.74 (14-C), 61.94, 55.83, 50.97, $49.58\left(2 \times \mathrm{CH}_{2}\right), 49.03,43.86,43.79,38.41,37.97,37.15,35.10$, $27.49,27.42,26.02\left(2 \times \mathrm{CH}_{2}\right), 25.48,24.54,22.09,21.86,21.33,20.19,19.46,19.13,15.07$. HRMS (ESI) calcd. for $\mathrm{C}_{30} \mathrm{H}_{50} \mathrm{~N}_{3} \mathrm{O}[\mathrm{M}+\mathrm{H}]^{+}: 468.3954$, found: 468.3938 .

N-[(1R,4aR,4bS,10aR)-1,4a-Dimethyl-7-(propan-2-ylidene)-1,2,3,4,4a,4b,5,6,7,9,10,10adodecahydrophenanthren-1-yl)-4-(dimethylamino)piperidine-1-carboxamide (8), Yield 73\%, colorless oil. ${ }^{1} \mathrm{H}-\mathrm{NMR}\left(400 \mathrm{MHz}, \mathrm{DMSO}-d_{6}\right) \delta 6.16(\mathrm{~s}, 1 \mathrm{H}, 14-\mathrm{CH}), 5.38(\mathrm{~s}, 1 \mathrm{H}, \mathrm{NH}), 3.89(\mathrm{~d}, J=13.1 \mathrm{~Hz}$, $2 \mathrm{H}), 2.63-2.51(\mathrm{~m}, 3 \mathrm{H}), 2.48-2.42\left(\mathrm{~m}, 1 \mathrm{H}, 12-\mathrm{CH}_{2}\right), 2.36-2.27(\mathrm{~m}, 1 \mathrm{H}), 2.23-2.08(\mathrm{~m}, 10 \mathrm{H}$, including $\left.2 \times \mathrm{NCH}_{3}\right), 1.91-1.83(\mathrm{~m}, 1 \mathrm{H}), 1.82-1.55\left(\mathrm{~m}, 15 \mathrm{H}\right.$, including $\left.2 \times \mathrm{CH}_{3}\right), 1.31-1.17(\mathrm{~m}, 4 \mathrm{H}), 1.14(\mathrm{~s}, 3 \mathrm{H})$, 1.10-0.99 (m, 1H), 0.69 (s, 3H). ${ }^{13} \mathrm{C}-\mathrm{NMR}\left(100 \mathrm{MHz}, \mathrm{DMSO}-d_{6}\right) \delta 156.53$ (18-CO), 138.51 (8-C), 127.84 (13-C), 122.47 (15-C), 121.73 (14-C), 61.56, 55.83, 50.98, 49.02, 43.46, 43.35, 41.26 (2 $\left.\times \mathrm{CH}_{3}\right), 38.41,37.98$, 37.16, 35.11, 27.91, 27.82, 25.48, 22.09, 21.86, 21.34, 20.20, 19.46, 19.14, 15.08. HRMS (ESI) calcd. for $\mathrm{C}_{27} \mathrm{H}_{46} \mathrm{~N}_{3} \mathrm{O}[\mathrm{M}+\mathrm{H}]^{+}:$428.3641, found: 428.3626 . 
$N-[(1 R, 4 a R, 4 b S, 10 a R)-1,4 a-D i m e t h y l-7-($ propan-2-ylidene)-1,2,3,4,4a,4b,5,6,7,9,10,10a-

dodecahydrophenanthren-1-yl]-4-morpholinopiperidine-1-carboxamide (9), Yield 69\%, colorless oil. ${ }^{1} \mathrm{H}-\mathrm{NMR}\left(500 \mathrm{MHz}\right.$, DMSO- $\left.d_{6}\right) \delta 6.17(\mathrm{~s}, 1 \mathrm{H}), 5.40(\mathrm{~s}, 1 \mathrm{H}), 3.90(\mathrm{~d}, J=13.1 \mathrm{~Hz}, 2 \mathrm{H}), 3.55(\mathrm{t}, J=4.5 \mathrm{~Hz}$, $4 \mathrm{H}), 2.62-2.52(\mathrm{~m}, 2 \mathrm{H}), 2.48-2.40(\mathrm{~m}, 4 \mathrm{H}), 2.32(\mathrm{dd}, J=14.4,4.1 \mathrm{~Hz}, 1 \mathrm{H}), 2.27-2.22(\mathrm{~m}, 1 \mathrm{H})$, $2.19(\mathrm{dd}, J=12.4,2.4 \mathrm{~Hz}, 1 \mathrm{H}), 2.15-2.05(\mathrm{~m}, 2 \mathrm{H}), 1.87(\mathrm{t}, J=7.5 \mathrm{~Hz}, 1 \mathrm{H}), 1.83-1.55(\mathrm{~m}, 13 \mathrm{H}$, including $\left.2 \times \mathrm{CH}_{3}\right), 1.52-1.36(\mathrm{~m}, 2 \mathrm{H}), 1.32-1.17(\mathrm{~m}, 4 \mathrm{H}), 1.14(\mathrm{~s}, 3 \mathrm{H}), 1.06(\mathrm{td}, J=13.2,3.8 \mathrm{~Hz}, 2 \mathrm{H})$, $0.69(\mathrm{~s}, 3 \mathrm{H}) .{ }^{13} \mathrm{C}-\mathrm{NMR}\left(125 \mathrm{MHz}, \mathrm{DMSO}-d_{6}\right) \delta 156.50$ (18-CO), 138.47 (8-C), 127.82 (13-C), 122.39 (15-C), 121.68 (14-C), $66.53\left(2 \times \mathrm{CH}_{2}\right), 61.31,55.81,50.95,49.30\left(2 \times \mathrm{CH}_{2}\right), 49.09,43.39,43.35,38.39,37.95$, $37.18,35.08,27.76,27.73,25.45,22.06,21.83,21.25,20.14,19.42,19.10,15.02$. HRMS (ESI) calcd. for $\mathrm{C}_{29} \mathrm{H}_{48} \mathrm{~N}_{3} \mathrm{O}_{2}[\mathrm{M}+\mathrm{H}]^{+}:$470.37410, found: 470.37415 .

1-[(1R,4aR,4bS,10aR)-1,4a-Dimethyl-7-(propan-2-ylidene)-1,2,3,4,4a,4b,5,6,7,9,10,10a-

dodecahydrophenanthren-1-yl]-3-(2-hydroxyphenyl)urea (10), Yield 70\%, white solid. ${ }^{1} \mathrm{H}-\mathrm{NMR}$ $\left(500 \mathrm{MHz}, \mathrm{CDCl}_{3}\right) \delta 9.64(\mathrm{~s}, 1 \mathrm{H}), 7.05-6.92(\mathrm{~m}, 2 \mathrm{H}), 6.89-6.82(\mathrm{~m}, 2 \mathrm{H}), 6.79(\mathrm{t}, J=7.5 \mathrm{~Hz}, 1 \mathrm{H}), 6.19$ $(\mathrm{s}, 1 \mathrm{H}), 5.12(\mathrm{~s}, 1 \mathrm{H}), 2.51(\mathrm{dt}, J=14.1,4.2 \mathrm{~Hz}, 1 \mathrm{H}), 2.39-2.31(\mathrm{~m}, 1 \mathrm{H}), 2.20-2.10(\mathrm{~m}, 1 \mathrm{H}), 2.04-1.98$ $(\mathrm{m}, 2 \mathrm{H}), 1.93-1.79(\mathrm{~m}, 3 \mathrm{H}), 1.74(\mathrm{~s}, 3 \mathrm{H}), 1.70(\mathrm{~s}, 3 \mathrm{H}), 1.65(\mathrm{~d}, J=12.9 \mathrm{~Hz}, 1 \mathrm{H}), 1.62-1.52(\mathrm{~m}, 2 \mathrm{H})$, $1.48-1.27(\mathrm{~m}, 4 \mathrm{H}), 1.25(\mathrm{~s}, 3 \mathrm{H}), 1.10(\mathrm{td}, J=13.2,3.7 \mathrm{~Hz}, 1 \mathrm{H}), 0.75(\mathrm{~s}, 3 \mathrm{H}) .{ }^{13} \mathrm{C}-\mathrm{NMR}\left(125 \mathrm{MHz}, \mathrm{CDCl}_{3}\right) \delta$ 156.69 (18-CO), 148.71, 138.30 (8-C), 128.39 (13-C), 126.76, 125.91, 123.65 (15-C), 122.53 (14-C), 122.37, $120.62,119.10,57.73,51.46,51.43,38.98,38.33,38.03,35.68,25.96,22.63,22.50,21.59,20.49,19.83,19.51$, 15.42. HRMS (ESI) calcd. for $\mathrm{C}_{26} \mathrm{H}_{37} \mathrm{~N}_{2} \mathrm{O}[\mathrm{M}+\mathrm{H}]^{+}: 409.28495$, found: 409.28506 .

1-[2-(1H-Indol-3-yl)ethyl)-3-((1R,4aR,4bS,10aR)-1,4a-dimethyl-7-(propan-2-ylidene)1,2,3,4,4a,4b,5,6,7,9,10,10a-dodecahydrophenanthren-1-yl)urea (11), Yield 89\%, white solid. ${ }^{1} \mathrm{H}-\mathrm{NMR}$ $\left(500 \mathrm{MHz}, \mathrm{CDCl}_{3}\right) \delta 8.34(\mathrm{~s}, 1 \mathrm{H}), 7.59(\mathrm{~d}, J=7.9 \mathrm{~Hz}, 1 \mathrm{H}), 7.36(\mathrm{~d}, J=8.1 \mathrm{~Hz}, 1 \mathrm{H}), 7.19(\mathrm{t}, J=7.4 \mathrm{~Hz}$, $1 \mathrm{H}), 7.11(\mathrm{t}, J=7.5 \mathrm{~Hz}, 1 \mathrm{H}), 6.99(\mathrm{~d}, J=2.4 \mathrm{~Hz}, 1 \mathrm{H}), 6.19(\mathrm{~s}, 1 \mathrm{H}), 4.33(\mathrm{~s}, 1 \mathrm{H}), 4.05(\mathrm{~d}, J=2.8 \mathrm{~Hz}, 1 \mathrm{H})$, $3.43(\mathrm{q}, J=6.2 \mathrm{~Hz}, 2 \mathrm{H}), 2.92(\mathrm{t}, J=6.7 \mathrm{~Hz}, 2 \mathrm{H}), 2.51(\mathrm{dt}, J=14.3,4.3 \mathrm{~Hz}, 1 \mathrm{H}), 2.39-2.31(\mathrm{~m}, 1 \mathrm{H}), 2.17$ $(\mathrm{t}, J=14.8 \mathrm{~Hz}, 1 \mathrm{H}), 2.04(\mathrm{td}, J=13.6,13.1,4.0 \mathrm{~Hz}, 1 \mathrm{H}), 1.97-1.80(\mathrm{~m}, 4 \mathrm{H}), 1.76(\mathrm{~s}, 3 \mathrm{H}), 1.70(\mathrm{~s}, 3 \mathrm{H})$, $1.64(\mathrm{~d}, J=13.1 \mathrm{~Hz}, 1 \mathrm{H}), 1.55(\mathrm{ddd}, J=17.0,9.8,4.3 \mathrm{~Hz}, 2 \mathrm{H}), 1.47-1.21(\mathrm{~m}, 4 \mathrm{H}), 1.15(\mathrm{~s}, 3 \mathrm{H}) 1.14-1.06$ (m, 1H), 0.73 (s, 3H). ${ }^{13} \mathrm{C}-\mathrm{NMR}\left(125 \mathrm{MHz}, \mathrm{CDCl}_{3}\right) \delta$ 157.26, 138.75, 136.56, 128.48, 127.62, 123.46, $122.37,122.23,122.17,119.54,118.91,113.45,111.43,56.63,51.45,51.25,40.97,38.97,38.44,38.19,35.78$, $26.17,26.01,22.65,22.39,22.02,20.48,19.82,19.65,15.43$. HRMS (ESI) calcd. for $\mathrm{C}_{30} \mathrm{H}_{42} \mathrm{~N}_{3} \mathrm{O}[\mathrm{M}+\mathrm{H}]^{+}$: 460.33279 , found: 460.33439 .

\section{Conclusions}

In summary, by application of Curtius rearrangement reaction, we designed and synthesized eleven new ureido derivatives of neoabietic acid. The general protocol was to obtain the isocyanate intermediate at first, then without isolation, it was then reacted with amine in a one-pot model. The reaction works smoothly to a broad range of aliphatic primary and secondary amine but is unfavorable to aromatic amines with electron-withdrawing groups. More ureido derivatives of neoabietic acid are under preparation and their biological activities will be reported in due course.

Supplementary Materials: ${ }^{1} \mathrm{H}-\mathrm{NMR},{ }^{13} \mathrm{C}-\mathrm{NMR}$ spectra of compounds 1-11 are available online.

Author Contributions: G.H. and J.C. initiated and supervised the project. X.G. and N.F. did the synthesis. Y.Z. helped in the purification and analysis of the compounds. G.H. and X.G. wrote the paper. All the authors gave their final approval for the publication.

Funding: This publication was supported by a grant from Shanghai Normal University (DYL201702).

Conflicts of Interest: The authors declare no conflict of interest. 


\section{References}

1. Keeling, C.I.; Bohlmann, J. Diterpene resin acids in conifers. Phytochemistry 2006, 67, 2415-2423. [CrossRef] [PubMed]

2. Park, J.Y.; Lee, Y.K.; Lee, D.S.; Yoo, J.E.; Shin, M.S.; Yamabe, N.; Kim, S.N.; Lee, S.; Kim, K.H.; Lee, H.J.; et al. Abietic acid isolated from pine resin (Resina Pini) enhances angiogenesis in HUVECs and accelerates cutaneous wound healing in mice. J. Ethnopharmacol. 2017, 203, 279-287. [CrossRef] [PubMed]

3. Hjortness, M.K.; Riccardi, L.; Hongdusit, A.; Ruppe, A.; Zhao, M.; Kim, E.Y.; Zwart, P.H.; Sankaran, B.; Arthanari, H.; Sousa, M.C.; et al. Abietane-type diterpenoids inhibit protein tyrosine phosphatases by stabilizing an inactive enzyme conformation. Biochemistry 2018, 57, 5886-5896. [CrossRef] [PubMed]

4. Yoshioka, H.; Mizuno, Y.; Yamaguchi, T.; Ichimaru, Y.; Takeya, K.; Hitotsuyanagi, Y.; Nonogaki, T.; Aoyagi, Y. Methyl dehydroabietate counters high fat diet-induced insulin resistance and hepatic steatosis by modulating peroxisome proliferator-activated receptor signaling in mice. Biomed. Pharmacother. 2018, 99, $214-219$. [CrossRef] [PubMed]

5. González, M.A. Aromatic abietane diterpenoids: Their biological activity and synthesis. Nat. Prod. Rep. 2015, 32, 684-704. [CrossRef] [PubMed]

6. Hou, W.; Zhang, G.; Luo, Z.; Li, D.; Ruan, H.; Ruan, B.H.; Su, L.; Xu, H. Identification of a diverse synthetic abietane diterpenoid library and insight into the structure-activity relationships for antibacterial activity. Bioorg. Med. Chem. Lett. 2017, 27, 5382-5386. [CrossRef] [PubMed]

7. Yang, Z.; Kitano, Y.; Chiba, K.; Shibata, N.; Kurokawa, H.; Doi, Y.; Arakawa, Y.; Tada, M. Synthesis of variously oxidized abietane diterpenes and their antibacterial activities against MRSA and VRE. Bioorg. Med. Chem. 2001, 9, 347-356. [CrossRef]

8. Lee, H.-J.; Ravn, M.M.; Coates, R.M. Synthesis and characterization of abietadiene, levopimaradiene, palustradiene, and neoabietadiene: Hydrocarbon precursors of the abietane diterpene resin acids. Tetrahedron 2001, 57, 6155-6167. [CrossRef]

9. Alvarez-Manzaneda, E.J.; Chahboun, R.; Guardia, J.J.; Lachkar, M.; Dahdouh, A.; Lara, A.; Messouri, I. New route to 15-hydroxydehydroabietic acid derivatives: Application to the first synthesis of some bioactive abietane and nor-abietane type terpenoids. Tetrahedron Lett. 2006, 47, 2577-2580. [CrossRef]

10. Lang, S.; Murphy, J.A. Azide rearrangements in electron-deficient systems. Chem. Soc. Rev. 2006, 35, $146-156$. [CrossRef] [PubMed]

11. Lin, S.J.; Su, T.C.; Chu, C.N.; Chang, Y.C.; Yang, L.M.; Kuo, Y.C.; Huang, T.J. Synthesis of C-4-substituted steviol derivatives and their inhibitory effects against hepatitis B Virus. J. Nat. Prod. 2016, 79, 3057-3064. [CrossRef] [PubMed]

(C) 2018 by the authors. Licensee MDPI, Basel, Switzerland. This article is an open access article distributed under the terms and conditions of the Creative Commons Attribution (CC BY) license (http://creativecommons.org/licenses/by/4.0/). 